\begin{tabular}{|c|c|c|c|c|c|c|c|c|c|c|c|}
\hline Epoch & Position & Nights & Dist. & Nights & Mean power & Magn. & \multicolumn{5}{|c|}{ Telescope in use. } \\
\hline 1839,66 & $27^{\circ} 42$ & 2 & $1{ }^{\prime \prime} 5 \pm$ & 1 & 320 & $2 \frac{1}{2}, 8 \frac{3}{4}$ & Mr. Bishop's & 7 -inch & refractor & by $J$ & Dollond. \\
\hline 40,67 & 25,19 & 2 & & & 370 & 3,9 & $=$ & $=$ & $=$ & $=$ & $=$ \\
\hline 41,89 & 23,75 & 4 & & & 416 & 3,9 & $\because$ & $=$ & $=$ & $=$ & $=$ \\
\hline 41,96 & & & 1,667 & 1 & 420 & 3,9 & $=$ & $=$ & $=$ & $=$ & $=$ \\
\hline 47,39 & 16,70 & 3 & & & 413 & $3,8 \frac{1}{4}$ & $\mathbf{M y} 6 \frac{1}{3}$ inch & by Merz & & & \\
\hline 48,75 & 14,53 & 3 & 1,767 & 3 & 417 & 3,8 & $=$ & $=$ & & & \\
\hline 51,51 & 11,56 & 2 & 1,652 & 2 & 546 & 3,8 & $=$ & $=$ & & & \\
\hline 52,74 & 10,78 & 2 & 1,683 & 2 & 375 & & $=$ & $=$ & & & \\
\hline 53,73 & 7,33 & 3 & 1,768 & 3 & 467 & 3,8 & $=$ & $=$ & & & \\
\hline 54,64 & 4,30 & 1 & 1,688 & 1 & 630 & & $7 \frac{1}{2}$-inch by & Alvan $C$ & Yark. & & \\
\hline 59,58 & 357,72 & 3 & 1,675 & 3 & 532 & $3.4,8$ & $8 \frac{\pi}{4}-$ inch $=$ & $=$ & $=$ & & \\
\hline 65,38 & 349,62 & 2 & $1,67 j$ & 2 & 492 & 3,8 & 8 -inch by & Cooke. & & & \\
\hline
\end{tabular}

A fine state of atmosphere was usually present when these measures were obtained, and on several occasions the smaller star was so well seen that the observations were by no means difficult. An excellent filar micrometer hy Dollond was used in every instance except in the year 1847.

The accuracy of the results for the present year is well supported by some measures which Mr. G. Knott has made with his excellent $7 \frac{1}{3}$-inch refractor by Alvan Clark; his results being : Pos. $=349^{\circ} 00 ;$ Dist. $=1^{\prime \prime} 705 ;$ Fpoch 1865,44 .

It seems necessarily to follow from this, that the continued diminution of distance indicated by some of the later observations, employed by Herrn Bchrmann in his computations, probably arose from erroneous measurement. Cerfain

it is, that if the central distance of the small star were now what is indicated by the Ephemeris, it could scarcely be separated from the larger star even by the Poulkowa refractor; whereas, in fact, it may be well seen with an aperture of 6 or $6 \frac{1}{4}$ inches (Engl.), and measured witbout difficulty with 7 inches on a suitable night.

Having always seen the smaller star with as much ease as night reasonably be expected under such circumstances as would induce me to measure it, I have never from $\mathrm{my}$ own observations suspected its brightness to be variable.

Hopefield Observatory, Haddenhaw, near Thame. Sept. 1865.

W. R. Dawes.

\title{
Observations de la planète (84) Clio à l'Observatoire Royal de Copenhague.
}

Mr. d'Arrest a obtenu les positions suivantes de la nouvelle planète :

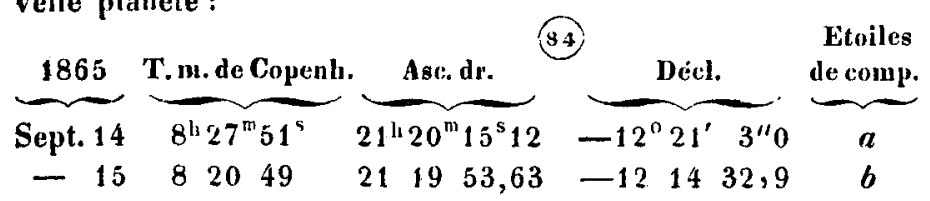

Depuis j'ai poursuivi la planète avec la grande lunette dans les belles soirées de Septembre, comme suit:

$\begin{array}{rrrrrrrrrrr}\text { Sept. } 18 & 8^{\mathrm{h}} 34^{\mathrm{m}} 34^{\mathrm{s}} & 21^{\mathrm{l}} 19^{\mathrm{m}} & 4^{\mathrm{s}} 38 & -11^{\circ} 54^{\mathrm{c}} 36^{\prime \prime} 1 & c \\ 19 & 8 & 16 & 36 & 21 & 18 & 52,94 & -11 & 48 & 8,9 & = \\ 20 & 8 & 9 & 35 & 21 & 18 & 43,38 & -11 & 41 & 31,6 & = \\ 21 & 8 & 7 & 47 & 21 & 18 & 37,04 & -11 & 34 & 37,2 & d \\ 22 & 8 & 22 & 54 & 21 & 18 & 32,47 & -11 & 27 & : & \\ 23 & 8 & 24 & 23 & 21 & 18 & 30,46 & -11 & 20 & 55,1 & = \\ 25 & 8 & 46 & 20 & 21 & 18 & 34,42 & -11 & 7 & 28,5 & \\ 26 & 8 & 21 & 8 & 21 & 18 & 40,13 & -11 & 0 & 39,0 & = \\ 27 & 9 & 16 & 31 & 21 & 18 & 47,60 & -10 & 53 & 26,1 & =\end{array}$

Sept. 18 la planète de $10^{\mathrm{me}}$ grandeur,

= 22 la planète très-faible, épais brouillard.

$=27$ éclat d'une étoile de $11^{\mathrm{m}} 3$ grandeur.

Voici les étoiles de comparaison, déterminées récemment au cercle méridien par Mr. Schjellerup, qui y a joint quelques autres observations d'étoiles situées dans la route de la pla- nète et employécs par d'autres astronomes dans leurs premières observations après la déconverte de Clio.

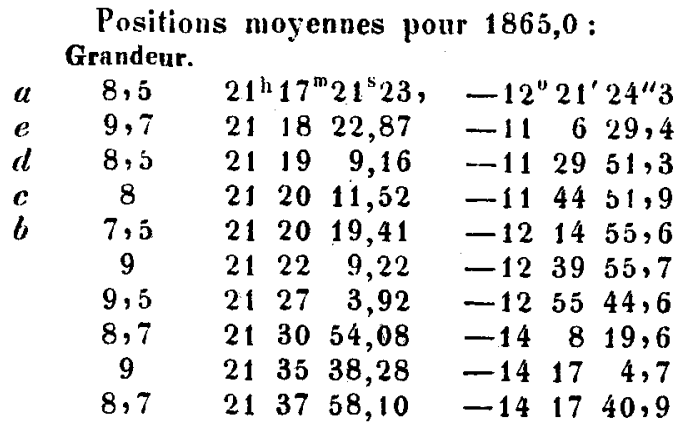

Mr. $d$ 'Arrest a encore obtenu une position de la comète périodique de Faye:

$$
\frac{1865}{\text { Sept. } 14} \cdot \underbrace{\text { T. m. Cop. }}_{8^{\mathrm{h}} 53^{\mathrm{m}} 2^{\mathrm{s}}} \underbrace{\text { Asc. dr. of }}_{21^{\mathrm{h}} 46^{\mathrm{m}} 6^{\mathrm{s}} 73} \underbrace{\text { Décl. of }}_{+2^{\circ} 52^{\prime} 27^{\prime \prime} 4}
$$

Aussi cette fois l'étoile de comparaison a été déterminée au cercle méridien. Cette position, comme toutes les précédentes, doit encore être corrigée par le moyen de la paralIaxe, mais elles sunt toutes corrigées de la réfraction.

Copenhague, 1865 Oct. 5.
Mynster-Fischer. 